\title{
Cytogenetics at the University of Cape Town: A 45-year journey
}

\author{
R D Smart, ${ }^{1-3}$ NDip, BSc Med (Hon); G Schutte, ${ }^{2,3,5}$ NDip, BTech; T Ruppelt, ${ }^{3-5}$ NDip, BTech; L J Greenberg, ${ }^{1-4,6} \mathrm{PhD}$ \\ ${ }^{1}$ Department of Physiology, Faculty of Medicine, University of Cape Town, South Africa \\ ${ }^{2}$ Department of Human Genetics, Faculty of Health Sciences, University of Cape Town, South Africa \\ ${ }^{3}$ Division of Human Genetics, Department of Clinical Laboratory Sciences, Faculty of Health Sciences, University of Cape Town, South Africa \\ ${ }^{4}$ Division of Human Genetics, Department of Pathology, Institute of Infectious Disease and Molecular Medicine, Faculty of Health Sciences, \\ University of Cape Town, South Africa \\ ${ }^{5}$ Cytogenetic Laboratory, National Health Laboratory Service, Groote Schuur Hospital, Cape Town, South Africa \\ ${ }^{6}$ Genetics Laboratory, National Health Laboratory Service, Groote Schuur Hospital, Cape Town, South Africa
}

Corresponding author: R D Smart (smarties@vinet.co.za)

This article is a brief record of the cytogenetics laboratory from its birth in 1971, under the auspices of the University of Cape Town, throughout its development within the Department of Human Genetics, under the leadership of Professor Peter Beighton, to its present position at Groote Schuur Hospital, as a multidisciplinary unit run by the National Health Laboratory Service.

S Afr Med J 2016;106(6 Suppl 1):S29-S32. DOI:10.7196/SAMJ.2016.v106i6.10987

\section{Phase one: The early laboratory}

Historically, the first laboratory discipline that most human genetics departments introduce has been a cytogenetic laboratory. Although this technically did happen in Cape Town at the University of Cape Town (UCT), there was a slight variation in the timing of this procedure as the laboratory preceded the department. In 1970, a small cytogenetics laboratory was opened in the Department of Physiology, under the auspices of Dr Pauline Webb, when Prof. A W Sloan was the head of department (HOD). In January 1971, Jacquie Greenberg (JG), a recent graduate from Stellenbosch University, was appointed as a temporary lecturer for 6 months as a locum for Pauline Webb who went on sabbatical leave. JG took over the oversight of the laboratory where Penny Kemp, a technologist from England, did the tissue culturing and karyotyping. JG, a full Professor at UCT from 2009, remained in the Faculty of Health Sciences (FHS) at UCT until her retirement in 2015. In August 1971, Ronald Smart (RS) from the University of Edinburgh was employed to formally activate the diagnostic laboratory with a view to offering a laboratory service to the local hospitals in the Western Cape.

In those early days, the laboratory was housed in one room in the basement of the physiology building (officially known as Wernher \& Beit South from 1925) ${ }^{[1]}$ and outside the window, which was often open, lived a large number of the genus Xenopus (African clawed frog) used for pregnancy testing - a technique developed by H Zwarenstein at UCT in 1934. ${ }^{[2]}$ This one room was used for setting up blood cultures, harvesting the cells to produce chromosomes, microscopic analysis and karyotyping. There were also a number of dassies caged nearby, and on one occasion a dassie made its escape into the dark room where photographs were developed for karyotyping. Needless to say, contamination was an ongoing problem! Those were interesting times. In April 1972, Peter Beighton (PB) was appointed as the first Professor of Human Genetics at $\mathrm{UCT}^{[3]}$ and the cytogenetics unit was incorporated into this new department, and housed in the then New Anatomy Building. The new laboratory had sealed sterile rooms, a dark room as well as a microscope and karyotyping area. At this stage the staff complement increased and routine chromosome analysis on blood samples was introduced. Later in 1972, Dr Molly Nelson (MN), a paediatrician with a special interest in clinical genetics, also from the University of Edinburgh, joined the department and was involved in the running of the cytogenetic section. At this point the laboratory was able to cope with the blood samples being sent for chromosome analysis and it was possible for RS to accompany MN on a regular basis to the various neonatal and paediatric wards. In this way a firsthand comprehensive knowledge of dysmorphology was obtained by the laboratory staff.

In those early days, the laboratory introduced culture and analysis of amniotic fluid samples. It is difficult to imagine that in the first year only 20 such samples were received. It is also of historical note that many samples were taken at that time without the use of ultrasound guidance. As a result, about one-third of the samples were contaminated with blood. A large number of these failed to grow successfully but with the introduction of ultrasound, the number of clear samples increased to almost $100 \%$, and the culture success rate rose accordingly. When the screening of chromosomes obtained from amniotic fluid was first started, a method using quinacrine stain was used - a fluorescent staining method that could identify the $Y$ chromosome. The routine Giemsa staining at this stage was prior to $\mathrm{G}$ banding and it was useful, and at times reassuring, to know whether the fetus was male or female. As this was a quick and simple test, the method was used routinely for many years. In addition, a service for cytogenetic analysis of skin and other tissue types was introduced. These were normally sent to confirm a previous blood diagnosis, or those from fetal tissue where a chromosome abnormality was suspected.

It was in this era that Prof. Peter Jacobs, the then Head of Haematology, approached the department to have a cytogenetic service available for some of his patients. The laboratory was able to begin this service immediately, mainly to detect the deletion in chromosome 22, known as the Philadelphia chromosome, often found in cases of chronic myeloid leukaemia. In addition, bone marrow (BM) cultures from patients who had undergone BM transplants were also established for further analysis. The idea was to look for markers in the chromosomes that would confirm that the transplant had been successful. If the donor was of a 
different sex to the recipient then it was a simple way to confirm the donor chromosomes. However, in same-sex donor/recipient patients, detection of small markers specific to the donor marrow chromosomes had to be established and relied upon.

It was decided at this stage to undertake a cytogenetic survey of the patients housed in the Alexandra Care and Rehabilitation Centre (formerly known as the Alexandra Institution). Accordingly, personnel from the laboratory visited the hospital each week and drew blood from five patients. Those samples were cultured and analysed and the results published some years later in 1982. ${ }^{[4]}$ Apart from the expected number of Down syndrome patients confirmed by chromosome studies, there were not a great many chromosome abnormalities in this population. However, it was noted that culturing of these bloods was problematic in that the number of metaphases obtained was low and the quality of the chromosomes was very poor indeed. This anomaly was present throughout the trial and in spite of attempts to improve the mitotic index, the situation was not remedied. It was not possible to explain why these cultures were so poor, compared with the samples set up at the same time under identical conditions from patients from other centres or hospitals.

\section{Phase two: Expansion}

In 1973, a biochemistry laboratory was opened in the same building, initially set up and run by JG. She was subsequently joined by Dr Howard Henderson, who transferred to the department from Red Cross War Memorial Children's Hospital (RCWMCH) chemical pathology laboratory in 1974.

In 1976, two individuals in South Africa (SA) were registered as Genetic Counsellors with the South African Medical and Dental Council. One was RS, working in the UCT laboratory and the other was Dr Stander Jansen, then head of the cytogenetic laboratory at University of the Free State. This opened up a novel combination for laboratory staff members to be involved in clinical service and was of great value when discussing laboratory findings directly with the patient. In this regard, and that of the earlier instances concerning the ward visits and subsequent teaching in dysmorphology, the UCT Department of Human Genetics was unique in the opportunity in which the laboratory staff found themselves. Prof. Beighton also organised weekly seminars that were open to all medical school staff. In addition, the department held weekly clinical meetings where staff from the laboratories, which now included the biochemistry section, and the clinical staff discussed cases, problems and future developments. As Prof. Beighton had a special interest in bone disorders, many of the presentations included an array of X-rays and all the staff members became well versed in viewing and commenting on the radiological abnormalities and anomalies to which they were exposed. In one case, a mosaic trisomy 8 was noted in a patient with broad clavicles. ${ }^{[5]}$ It cannot be stressed enough how important this clinical input, which PB encouraged in all its various forms, was to the laboratory staff and we were, and still are, grateful that the HOD placed so much weight on the clinical aspect of genetics.

In 1984, a molecular laboratory was opened next to the biochemistry laboratory, under the leadership of Dr Gillian Wallis. She was subsequently joined by JG, who then registered as a $\mathrm{PhD}$ student under GW and PB's supervision in 1987. The cytogenetic laboratory moved into a newly furbished adjoining laboratory and there can be no doubt that having the three laboratory disciplines housed together was a major advantage and the constant and regular contact with the clinical team was of great benefit to all concerned. The cytogenetic laboratory staff continued to grow and samples were sent for culture and analysis from as far away as the Eastern Cape and Namibia. In 1987, Dr Nelson retired and the laboratory was then run by RS.

During the 1980s, the laboratory purchased a semi-automated karyotyping system that allowed the chromosomes to be identified on a computer screen. Photographs were obtained digitally, directly from the microscopes, thereby alleviating the need for a dark room. The chromosome abnormality rate in blood samples remained constant at about $20 \%$ per annum and the amniotic fluid abnormalities detected ranged between $7 \%$ and $12 \%$. This was considerably more than some overseas centres and reflects the strict criteria imposed by the laboratory on patients being referred for chromosome analysis. In 1969, Lubs $^{[6]}$ described a condition of mental retardation among male members in one family. This so-called 'fragile X syndrome', which could only be detected using a modified culture technique, was later described by Sutherland. ${ }^{[7]}$ Laboratories worldwide re-examined earlier cases, usually when two or more male siblings were mentally retarded and had other physical signs of this newly discovered condition, but where previous chromosome studies on these individuals had been reported as normal. The UCT laboratory also recalled such patients, as did the other laboratories in SA. One large family ${ }^{[8]}$ and a small number of other families were identified. It was thought that this condition could be as common as Down syndrome, if not more prevalent. However, after the initial surge in referrals, it became apparent that this was not the case and these findings were subsequently reported. ${ }^{[9]}$ Contact with the other SA centres confirmed this observation. It then became possible to identify positive cases using molecular genetic techniques. However, the number of positive cases using this method remained low. When a positive case was identified, cytogenetic studies were also carried out and it was gratifying to note that the two methods always confirmed each other.

Around this time, many centres overseas had introduced chromosome analyses on chorion villi samples. The UCT laboratory did some work in this field and RS was afforded the opportunity to learn the technique at British Columbia Children's Hospital in Vancouver. Many of the potential referrals would have come from the advanced maternal age group, but many of those patients believed that they 'knew the ropes' and so did not present themselves early enough in pregnancy (ideally at $10-11$ weeks) for the test to be offered.

During the 1990s, there was considerable financial restraint placed on the health services in SA, which also impinged on the laboratories. In particular, if a staff member resigned, the post was not filled. Eventually some restrictions had to be enforced on the referral criteria. As amniotic fluid results are required urgently, the only way to report on those within an acceptable timeframe was to limit the intake from patients of advanced maternal age. The criteria were changed to a maternal age of 40 years and over, instead of the previous age of 35 years and over.

Ironically, in contrast to this limitation on the antenatal chromosome studies, a new technique known as fluorescence in situ hybridisation (FISH) was being introduced in many cytogenetic laboratories internationally. Trials were introduced and the UCT laboratory also began offering this FISH service. FISH is a combination of cytogenetic and molecular methodologies, where both metaphase and interphase cells can be analysed using whole chromosome paints (DNA probes) for the identification of translocations, centromere-specific probes for aneuploidy, and locus-specific probes for deletions, duplications and detection of gene rearrangements found in oncology-related conditions. Fluorochrome markers allow visualisation of the abnormalities using a fluorescent microscope. 
This technique provides a quick method for detecting the common trisomies - 21, 18 and 13 - without the need for culture. In addition to the use of FISH in antenatal diagnosis, the same method could be utilised for urgent confirmation of a clinical diagnosis in newborn babies, and this was also introduced on a routine basis. FISH studies in haematology disorders that had subtle chromosome abnormalities also increased dramatically. At this stage, the laboratory's range of tests still did not include haematological cytogenetics (including the new FISH studies) and those continued to be undertaken by the Department of Haematology.

While all this expansion was occurring, the laboratory moved yet again in 2003 to the Falmouth Building, FHS. At this stage, another change of great importance occurred with the introduction of the new National Health Laboratory Service (NHLS) being introduced nationwide. This resulted in a strengthening of the laboratory diagnostic services in general and allowed the cytogenetic laboratory to employ new staff including student medical technologists. Also, as a result of this new takeover there was collaboration between the UCT laboratory and the laboratory at Tygerberg Hospital, with the administration of the latter being taken over by RS although the laboratories continued to work independently.

At the end of 2006, a new laboratory was allocated space at Groote Schuur Hospital (GSH) and RS was able to have some input into the design of the new laboratory, although never having the opportunity to work in it as he retired as head of the laboratory; the position of head was taken over by GS, who had been involved with the department for many years working in the biochemistry and molecular sections, as well as cytogenetics. Subsequently GS was appointed by the NHLS as the Discipline Specific Laboratory Manager for Cytogenetics, which was still housed in the Falmouth Building.

GS attended the 9th course in Molecular Cytogenetics and DNA Microarrays in October 2007 (EGF University Bertinoro, Italy) with a view to preparing the laboratory to have amniotic fluid samples ready for testing when the molecular laboratory had the necessary filters for quantitative fluorescence-polymerase chain reaction (QF-PCR) analysis. ${ }^{[10]}$ Samples for haematology cytogenetics were sent from GSH and the private haematology units. All staff rotated through this area to become proficient in BM analysis.

\section{Phase three: The present laboratory}

In December 2007, the cytogenetics laboratory, consisting of five medical technologists and one student in cytogenetics, made the move from the FHS to the newly completed laboratory in GSH. This new NHLS section ${ }^{[11]}$ at GSH, called C21, housed a molecular genetics laboratory for haematology, chemistry, genetics, tissue immunology and the new cytogenetics karyotyping laboratory, incorporating FISH for acquired and constitutional conditions. With RS's retirement, the diagnostic laboratory, although academically connected with UCT, was then entirely run under the auspices of the NHLS and all cytogenetic staff members are now employed by the NHLS.

Once the move to the new laboratory had been completed, a number of major changes took place involving research and development of new techniques and significant changes to the staff complement. The haematology cytogeneticist who had been working in isolation in a haematology laboratory moved into the cytogenetics laboratory, bringing with her an additional station for the automatic karyotyping system, which had been installed in June 2007. The laboratory now had three karyotyping stations linked to a system that allowed capture of the chromosomes in metaphase, sorting on screen, with application of ideogram data and a print-out of the final karyotype with patient details. The inclusion of the BM haematology work into the laboratory enhanced the teaching requirements for medical technologists wanting to write the Medical Technology board exam for cytogenetics. Between December 2007 and May 2009, haematology FISH was established in the cytogenetics laboratory for haematological cancer identification and detection of minimal residual disease, in conjunction with polymerase chain reaction (PCR) technology done in the molecular genetics laboratory

The administration system used by the NHLS was adopted for logging and reporting samples. The inter-laboratory sample exchange that had been instituted in 2007 was replaced with an external quality control programme from the College of American Pathologists. All technologists were given the opportunity to analyse the challenge samples including karyotyping, aneuploidy, microdeletion and oncology FISH analysis. Now that the cytogenetics laboratory had a permanent home, application was made for South African National Accreditation System (SANAS) accreditation. The first assessment by SANAS was done in the cytogenetics laboratory in 2008 and the laboratory passed with flying colours. ${ }^{[12]}$

Investigations were also being undertaken to introduce faster molecular techniques for antenatal diagnosis. FISH for aneuploidy was well established, and samples from GSH and Tygerberg Hospital were being processed. The next, possibly cheaper, option - a quantitative PCR method, QF-PCR - was considered in collaboration with UCT.

When GS left the laboratory at the end of May 2009, there were seven qualified cytogenetic medical technologists, one student medical technologist and the cost centre supported one scientist and two genetic counsellors. SANAS had completed two successful assessments of the laboratory with no non-conformances. Detection rate of abnormal cytogenetics was running constantly at $20 \%$. GS was the academic advisor for the software development of the LabTrak Health Laboratory Information System. The managerial responsibility was handed over to Theresa Ruppelt (TR), an experienced senior technologist in the laboratory.

In 2009, TR was appointed to the post of Laboratory Manager. The first goal for the year was the consolidation of the Tygerberg Hospital (TBH) cytogenetics laboratory with the GSH laboratory. During September and October 2010, samples originating in TBH were sent to GSH, and the TBH cytogenetics laboratory closed in December 2010. These extra samples effectively doubled the workload. However, the turnaround time (TAT) remained the same. All FISH requests were already being done at GSH. Collaboration between the cytogenetic and molecular laboratories increased and cross-training of the cytogenetic technologists was accomplished. This was especially advantageous in light of the international trend towards molecular cytogenetic methods. One of the medical technologists in cytogenetics moved to the molecular laboratory on a permanent basis. All administrative duties were taken over from the technical staff by the appointment of a laboratory clerk in 2010. This assisted greatly in the improvement of the total TAT. There was an increase in requests for chromosome breakage studies for Fanconi's anaemia investigation and this test was validated for implementation in 2010.

Following the release of the consensus statement by Miller et al. ${ }^{[13]}$ recommending chromosomal microarray as the firsttier test in the diagnosis of developmental delay and multiple congenital abnormalities, a translational project was proposed for the diagnostic use of chromosomal microarray. The karyotyping system had a software update and an additional review station was installed in 2011. The new software improved the resolution of the karyotyping images. A further consolidation of cytogenetic services occurred in 2011 with the referral of all BM FISH and BM 
culture requests from $\mathrm{RCWMCH}$ to GSH towards the end of the year. QF-PCR was introduced into the diagnostic service by the end of 2011.

Supervisors were appointed on the BM, FISH, QF-PCR and amniotic fluid benches to improve on methodologies, TAT and to maintain consistency in training. The TAT of amniotic fluid cultures was less than 14 days. The demand for the introduction of more haematological FISH probes had resulted in the expansion of the probe inventory. A plasma cell labelling technique for multiple myeloma was introduced for a more accurate result. The need for the implementation of cytogenomic microarray was still shown to be of great importance. The feasibility study undertaken proved the efficacy of this technology through the results obtained. TR attended a 1-month training course at the Laboratory of Diagnostic Genomic Analysis in Leiden, Netherlands, in 2015.

\section{Conclusion}

In summary, the cytogenetics laboratory has evolved into a multifaceted, multi-tasking facility since the early days with the addition of new tests and an increase in sample volume and staff. The laboratory staff have always been involved in the training of various colleagues within the genetic services. The majority of the intern medical technologists passed the Cytogenetic Board Examination with distinction. As a SANAS accredited laboratory, the cytogenetics laboratory at GSH delivers a quality diagnostic service with an excellent TAT. Despite financial and staffing constraints the staff have shown perseverance and have focused on the task at hand. The future of cytogenetics at GSH is in line with the trend towards the next generation of technologies, which includes the continued use of high-definition chromosome analysis. Introduction of cytogenomic microarray into the diagnostic laboratory is the next goal for this laboratory.

\section{References}

1. Dent DM, Perez G. The place and the person: Named buildings, rooms and places on the campus of the Faculty of Health Sciences, University of Cape Town. S Afr Med J 2012;102(6):396-399.

2. Sloan AW. Highlights of physiological research at the University of Cape Town, 1912-1978. S Afr Med J 1979;56(22):974-979.

3. Beighton P, Fieggen K, Wonkam A, Ramesar R, Greenberg J. UCT's contribution to medical genetics in Africa - from the past into the future. S Afr Med J 2012;(102)6:446-448.

4. Nelson $\mathrm{MM}$, Smart RD. The results of chromosome examinations in an institution for mental 4. Nelson MM, Smart RD. The results of chromosome examinat
retardates in the Cape Province. S Afr Med J 1982;62(1):25-29.

5. Beighton P, Kozlowski KS, Gardner J, Smart RD. Broad clavicles in trisomy 8 mosaicism: A new sign. Skeletal Radiol 1999;28(6):359-361.

6. Lubs HA. A marker X chromosome. Am J Hum Genet 1969;21(3):231-244.

7. Sutherland GR. Marker X chromosomes and mental retardation. N Engl J Med 1977;296(24):1415.

8. Gardner RJ, Smart RD, Cornell JM, Merckel LM, Beighton P. The fragile X chromosome in a large Indian kindred. Clin Genet 1983;23(4):311-317.

9. Smart RD. Fragile X syndrome: As common as first thought? J Med Genet 1992;29(4):287.

10. Instituto de Estudios de Ciencias de la Salud de Cstilla y León. 9th Course in Molecular Cytogenetics and DNA Microarrays. Soria: IECSCYL; 2007

11. National Health Laboratory Service. NHLS. Available from: http://www.nhls.ac.za

12. South African National Accreditation Service. SANAS. Available from: http://home.sanas.co.za

13. Miller DT, Adam MP, Aradhya S, et al. Consensus statement: Chromosomal microarray is a first-tier

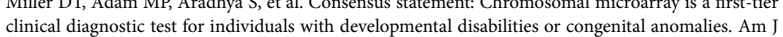
Hum Genet 2010;86(5):749-764. DOI:10.1016/j. aihg.2010.04.006 